\title{
Survival Benefit of Preoperative Versus Postoperative Radiotherapy in Metastatic Rectal Cancer Treated With Definitive Surgical Resection of Primary Tumor: A Population Based, Propensity Score-Matched Study
}

\author{
Dakui Luo ${ }^{1,3}$, Qi Liu'1,3, Ji Zhu²,3, Yanlei Ma1,3, Sanjun Cai1,3, Qingguo Li ${ }^{1,3}{ }^{凶}$, Xinxiang $\mathrm{Li}^{1,3 凶}$ \\ 1. Department of Colorectal Surgery, Fudan University Shanghai Cancer Center, Shanghai 200032, China \\ 2. Department of Radiation Oncology, Fudan University Shanghai Cancer Center, Shanghai 200032, China \\ 3. Department of Oncology, Shanghai Medical College, Fudan University, Shanghai 200032, China \\ Dakui Luo and Qi Liu contributed equally to this work.
}

$\square$ Corresponding authors: Xinxiang Li, 11491xx@sina.com; Qingguo Li, oncosurgeonli@sohu.com. No. 270, Dong'an Road, Xuhui District, Shanghai, China. Tel: +86021-64175590; Fax: +86021-64175590

(c) Ivyspring International Publisher. This is an open access article distributed under the terms of the Creative Commons Attribution (CC BY-NC) license (https://creativecommons.org/licenses/by-nc/4.0/). See http://ivyspring.com/terms for full terms and conditions.

Received: 2018.07.05; Accepted: 2018.12.14; Published: 2019.01.30

\begin{abstract}
Preoperative chemoradiation followed by surgery has been recommended as a standard treatment for patients with stage II/III rectal cancer. However, the optimal sequencing of radiotherapy for metastatic rectal cancer remains unclear. Between 2004 and 2014, patients diagnosed with metastatic rectal cancer who underwent the resection of primary site and received radiotherapy were retrospectively selected using the Surveillance, Epidemiology, and End Results (SEER) database. The propensity score matching analyses were used to lessen the effects of confounding factors including age, sex, race, marital status, serum carcinoembryonic antigen level, histologic type, differentiation status, tumor size, $\mathrm{T}$ stage, $\mathrm{N}$ stage and resection of the distant lesions. The cancer-specific survival (CSS) were compared based on the sequencing of radiotherapy. Ultimately, 686 matched pairs were formed for comparison of preoperative versus postoperative radiotherapy. The 5-year CSS estimates were $33.4 \%$ (95\% Cl: $28.9 \%-37.9 \%$ ) and $26.8 \%$ (95\% Cl: $22.7 \%-30.9 \%)$ for patients underwent preoperative radiotherapy followed by resection of primary lesion and postoperative radiotherapy after surgery, respectively. Patients underwent preoperative radiotherapy had better CSS as compared to patients received postoperative radiotherapy $(p<0.001$ for log-rank test). Multivariate analysis demonstrated that preoperative radiotherapy group was associated with significantly decreased risk for cancer death $(H R=0.820$, $95 \% \mathrm{Cl}: 0.712-0.945, \mathrm{p}=0.006$ ). Preoperative radiotherapy was superior to postoperative radiotherapy in patients with metastatic rectal cancer. Therapeutic strategy for these patients should be further explored.
\end{abstract}

Key words: neoadjuvant radiotherapy; metastatic rectal cancer; propensity score matching

\section{Introduction}

Over the past decades, the therapeutic strategy of radiation in the management of rectal cancer has evolved [1]. Preoperative versus postoperative radiotherapy for stage II/III rectal cancer has been well explored [2]. A prospective randomized controlled trial conducted by the German Rectal Cancer Study Group (the CAO/ARO/AIO-94 trial) demonstrated that preoperative chemoradiotherapy was associated with a decrease local recurrence and therapeutic toxicity as compared to postoperative chemoradiotherapy [3]. Furthermore, the advantage of local control in preoperative group persisted when it comes to 10-year cumulative incidence of local recurrence. It is regret that overall survival benefit was not observed in this study. In an earlier research, preoperative radiotherapy not only reduces rates of local recurrence but also improves survival among patients with resectable rectal cancer [4]. Besides that, preoperative radiation may result in tumor regression that increases the likelihood of sphincter preservation 
[5]. However, preoperative radiotherapy may be unnecessary due to exaggerated clinical stage [6]. Taking the advantages and disadvantages into account, the NCCN panel recommends preoperative chemoradiotherapy as a standard treatment for patients with stage II/III rectal cancer.

Due to the lack of randomized data for metastatic rectal resulted from complexity of stage IV disease, the therapeutic decisions are largely extrapolated from stage III rectal cancer. Postoperative adjuvant chemoradiotherapy is recommended for stage IV patients without receiving preoperative chemoradiotherapy or with a high risk for local recurrence after resection of primary lesion. However, the optimal sequencing of radiotherapy in metastatic rectal cancer remains unclear. To fill this gap in present guideline, we retrospectively analyzed data from Surveillance, Epidemiology, and End Results (SEER) database using propensity score matching analyses to determine if there are any survival benefits in patients with metastatic rectal cancer who were received neoadjuvant radiotherapy vs adjuvant radiotherapy.

\section{Material and methods}

\section{Data collection}

Patients with metastatic rectal cancer who underwent the resection of primary site and received preoperative and postoperative radiotherapy were identified in the SEER database (2004-2014). This study was approved by the Institutional Review Board of Fudan University Shanghai Cancer Center. The inclusion criteria were listed as follows: The site code represented "rectum (130)"; patients with distant metastases (M1); surgery was performed in primary site; patients with a known treatment sequence consisting of "radiation before surgery" (preoperative radiotherapy), "radiation after surgery" (postoperative radiotherapy); CRC was the only type of primary cancer; information about cancer-specific survival (CSS) and survival months were available.

The following data were collected: age at diagnosis, gender, marital status, race, serum CEA level, histologic type, differentiation status, tumor size, T stage, N stage, CSS, surgery of the metastases, radiation sequence with surgery. CSS was defined as the time from the date of diagnosis to the date of death caused by CRC. To minimize the inherent selection bias in a retrospective background, propensity score matching (PSM) at a 1:1 ratio was performed to compare CSS. The PSM model was based upon age, gender, marital status, race, serum CEA level, histologic type, differentiation status, tumor size, $\mathrm{T}$ stage, $\mathrm{N}$ stage and surgery of the metastases. The details of PSM process are demonstrated as Figure 1.

\section{Statistical analysis}

The differences between two groups were calculated by using $\chi^{2}$ test. The Kaplan-Meier method was used to analyze CSS and relative 5-year survival rate was calculated. The difference was compared using the log-rank test. Multivariate analyses were performed to recognize the specific factors which influence CSS. All statistical analyses were performed with SPSS 25.0 and R (version 3.4.4).

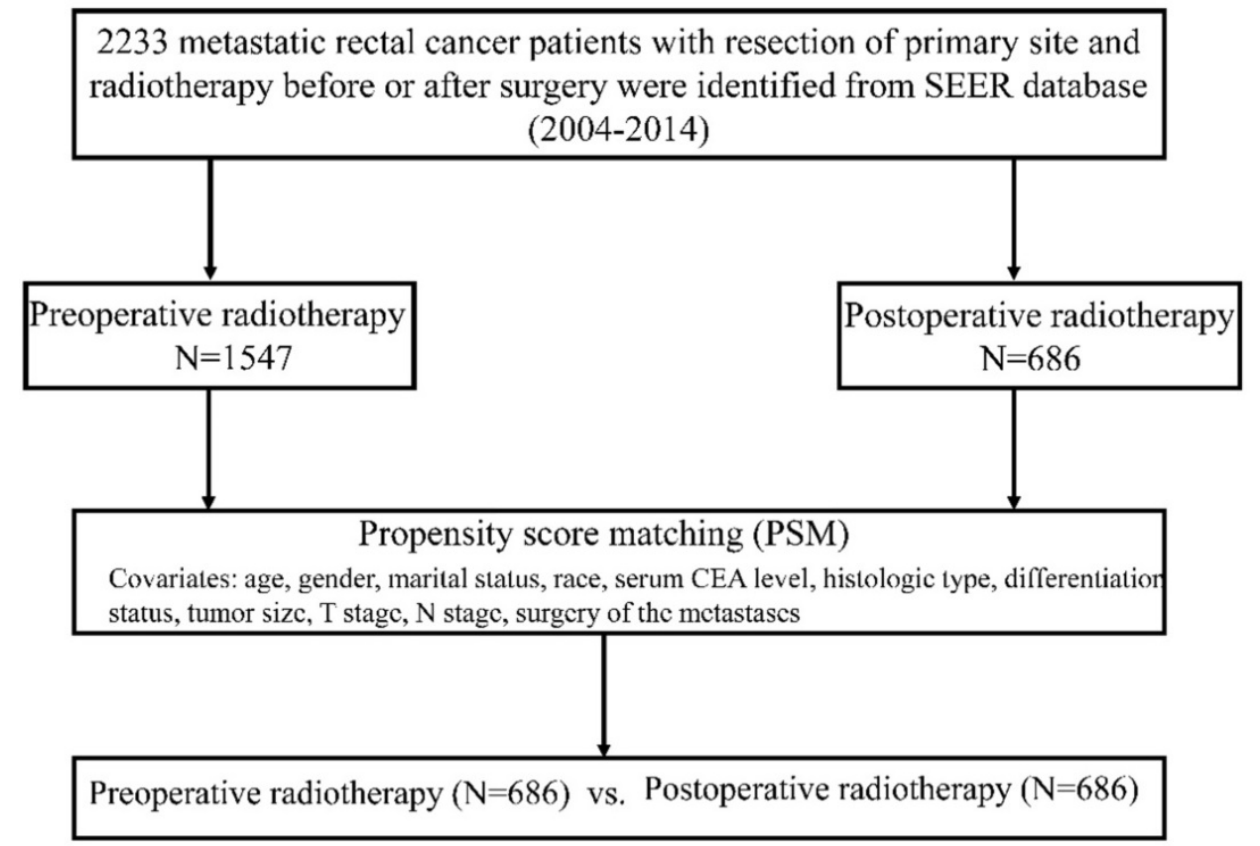

Figure 1. Flow diagram of the PSM process 
Table 1. Clinical characteristics of patients with metastatic patients receiving preoperative or postoperative radiotherapy.

\begin{tabular}{|c|c|c|c|c|c|}
\hline \multirow[t]{2}{*}{ Variable } & \multicolumn{2}{|c|}{$\begin{array}{l}\text { Radiation prior to rectal } \\
\text { surgery }\end{array}$} & \multicolumn{2}{|c|}{$\begin{array}{l}\text { Radiation after rectal } \\
\text { surgery }\end{array}$} & \multirow[t]{2}{*}{$P$ value } \\
\hline & Number & $\%$ & Number & $\%$ & \\
\hline Age & & & & & 0.550 \\
\hline$\leq 60$ & 387 & 50.7 & 376 & 49.3 & \\
\hline$>60$ & 299 & 49.1 & 310 & 50.9 & \\
\hline Sex & & & & & 0.869 \\
\hline Male & 406 & 50.2 & 403 & 49.8 & \\
\hline Female & 280 & 49.7 & 283 & 50.3 & \\
\hline Marital status & & & & & 0.219 \\
\hline Unmarried & 252 & 48.3 & 270 & 51.7 & \\
\hline Married & 421 & 51.7 & 393 & 48.3 & \\
\hline Unknown & 13 & 36.1 & 23 & 63.9 & \\
\hline Race & & & & & 0.389 \\
\hline White & 560 & 50.5 & 548 & 49.5 & \\
\hline Black & 59 & 44.4 & 74 & 55.6 & \\
\hline Others & 67 & 51.1 & 64 & 48.9 & \\
\hline Serum CEA & & & & & 0.024 \\
\hline Elevated & 336 & 57.0 & 353 & 43.0 & \\
\hline Normal & 146 & 48.8 & 110 & 51.2 & \\
\hline Unknown & 204 & 47.8 & 223 & 52.2 & \\
\hline Histologic type & & & & & 0.920 \\
\hline Adenocarcinoma & 515 & 50.2 & 511 & 49.8 & \\
\hline $\begin{array}{l}\text { Mucinous } \\
\text { adenocarcinoma }\end{array}$ & 71 & 50.0 & 71 & 50.0 & \\
\hline $\begin{array}{l}\text { Signet ring cell } \\
\text { carcinoma }\end{array}$ & 8 & 42.1 & 11 & 57.9 & \\
\hline Others & 92 & 49.7 & 93 & 50.3 & \\
\hline Differentiation & & & & & 0.082 \\
\hline Well & 29 & 49.2 & 30 & 50.8 & \\
\hline Moderate & 442 & 52.2 & 405 & 47.8 & \\
\hline $\begin{array}{l}\text { Poor and } \\
\text { undifferentiated }\end{array}$ & 154 & 45.0 & 188 & 55.0 & \\
\hline Unknown & 61 & 49.2 & 63 & 50.8 & \\
\hline Tumor size & & & & & 0.366 \\
\hline$<5 \mathrm{~cm}$ & 256 & 50.7 & 249 & 49.3 & \\
\hline$\geq 5 \mathrm{~cm}$ & 280 & 47.9 & 304 & 52.1 & \\
\hline Unknown & 150 & 53.0 & 133 & 47.0 & \\
\hline T stage & & & & & 0.055 \\
\hline $\mathrm{T} 1+\mathrm{T} 2$ & 56 & 42.7 & 75 & 57.3 & \\
\hline $\mathrm{T} 3+\mathrm{T} 4$ & 601 & 51.6 & 564 & 48.4 & \\
\hline Unknown & 29 & 38.2 & 47 & 61.8 & \\
\hline \multicolumn{6}{|l|}{ N stage } \\
\hline No & 125 & 46.0 & 147 & 54.0 & 0.089 \\
\hline $\mathrm{N}+$ & 533 & 51.7 & 497 & 48.3 & \\
\hline Unknown & 28 & 40.0 & 42 & 60.0 & \\
\hline $\begin{array}{l}\text { Surgery of } \\
\text { metastases }\end{array}$ & & & & & 0.280 \\
\hline No & 543 & 49.3 & 558 & 50.7 & \\
\hline Yes & 141 & 53.0 & 125 & 47.0 & \\
\hline Unknown & 2 & 40.0 & 3 & 60.0 & \\
\hline
\end{tabular}

\section{Results}

\section{Characteristics of patients}

A total of 2233 metastatic rectal cancer patients who underwent the resection of primary site and received radiotherapy with explicit treatment sequence were identified. After PSM, 686 matched pairs were recruited for comparison of preoperative versus postoperative radiotherapy. The clinicopathological characteristics of patients are comparable between the two groups and summarized in Table 1. Patients with elevated serum CEA level constituted a higher percentage in preoperative radiotherapy group than in the postoperative radiotherapy group. In terms of other variables, the percentage of patients was similar in two groups.

\section{Survival outcomes}

Median CSS for patients with preoperative and postoperative radiotherapy was 39 and 32 months, respectively. The Kaplan-Meier survival curves revealed a significant CSS advantage for preoperative radiotherapy ( $\mathrm{p}<0.001$ for log-rank test) (Figure 2A). The 5-year CSS estimates were $33.4 \% \quad(95 \%$ CI: $28.9 \%-37.9 \%$ ) and $26.8 \%$ (95\% CI: $22.7 \%-30.9 \%$ ) for patients underwent preoperative radiotherapy followed by resection of primary lesion and postoperative radiotherapy after surgery, respectively. The survival benefits were only observed in patients with adenocarcinoma instead of mucinous adenocarcinoma or signet ring cell carcinoma (Figure 2B-2D).

In multivariate analysis, age $>60$ year at diagnosis $(\mathrm{HR}=1.393,95 \% \mathrm{CI}: 1.211-1.603, \mathrm{P}<0.001)$ was associated with an increased risk of cancer death while married (HR=0.735, 95\% CI: 0.636-0.849, $\mathrm{P}<0.001)$, normal serum CEA level $(\mathrm{HR}=0626,95 \% \mathrm{CI}$ : $0.512-0.746, \mathrm{p}=<0.001$ ), preoperative radiotherapy $(\mathrm{HR}=0.820,95 \% \mathrm{CI}: 0.712-0.945, \mathrm{p}=0.006)$, and surgery of metastases (HR=0.606, 95\% CI: 0.498-0.736, $\mathrm{P}<0.001)$ resulted in decreased risk of cancer death (Table 2).

\section{Discussion}

Neoadjuvant chemoradiotherapy followed by total mesorectal excision has been the standard care for locally-advanced rectal cancer [7]. However, the therapeutic algorithm of radiotherapy in metastatic rectal cancer has been little investigated. Similarly, for resectable metastatic cases, the necessity of radiotherapy for local control has not been clarified.

Survival benefits of radiotherapy in metastatic rectal cancer have been shown in several retrospective studies [8,9]. However, another study found that postoperative pelvic radiotherapy did not improve recurrence-free survival of metastatic rectal patients after resection of primary tumor [10]. Indeed, stage IV rectal cancers comprise a heterogeneous group, which impedes the progress of prospective studies. Several studies with small sample size compared of local recurrence rate and overall survival of stage IV rectal cancer patients with or without neoadjuvant chemoradiotherapy and the results are inconsistent [11-14]. In the present study, we demonstrated that patients with resectable rectal cancer received radiotherapy before definitive primary resection surgery had better prognosis than radiotherapy after surgery using a relative large-population bases database. 

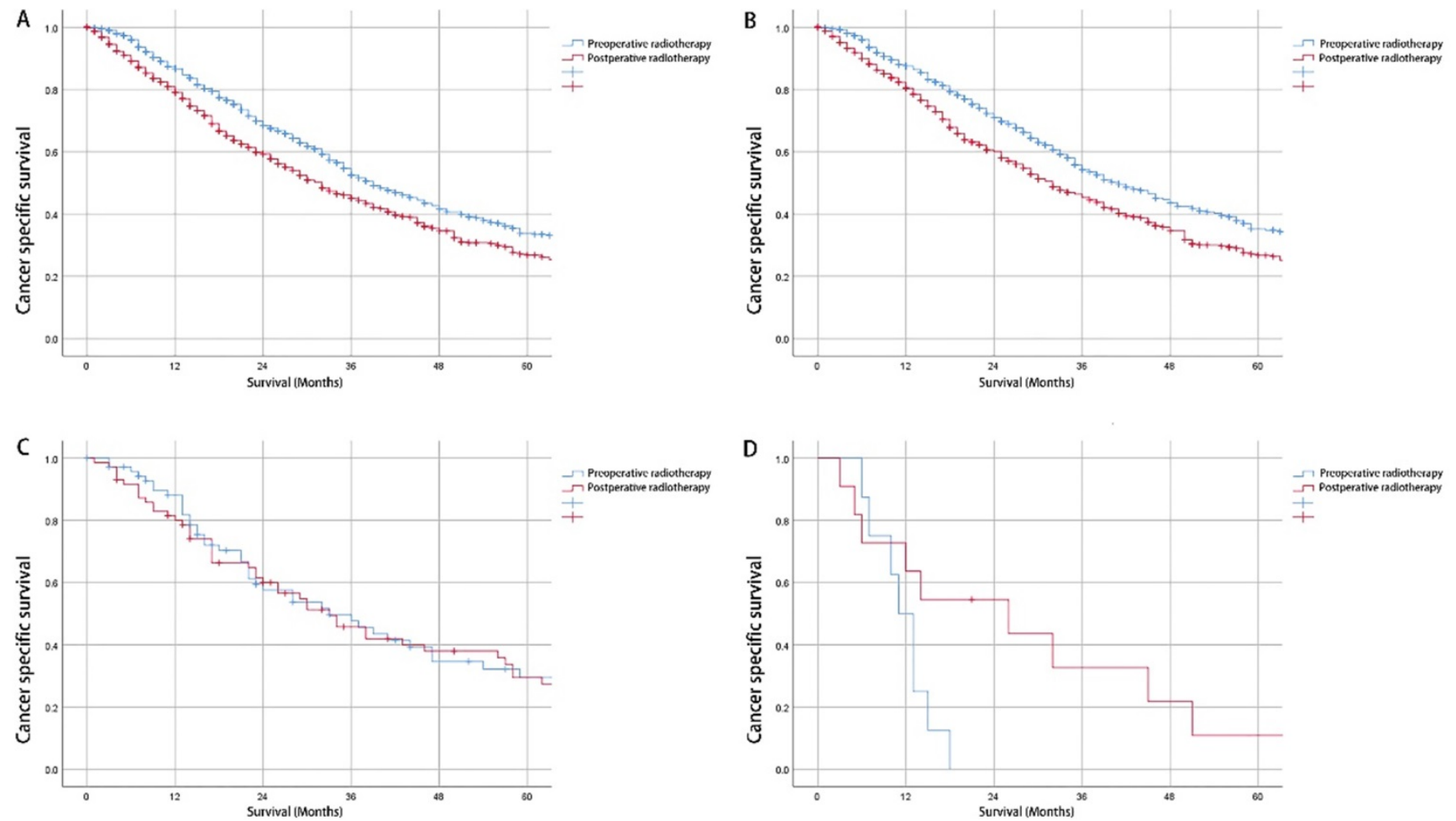

Figure 2. The Kaplan-Meier survival curves for preoperative and postoperative radiotherapy. A: All histological types cohort; B: Adenocarcinoma cohort; C: Mucinous adenocarcinoma cohort; D: Signet ring cell carcinoma cohort.

Table 2. Univariate and multivariate analysis for cancer-specific survival in metastatic rectal cancer with resection of primary site.

\begin{tabular}{|c|c|c|c|c|}
\hline \multirow[t]{2}{*}{ Variable } & \multicolumn{2}{|c|}{ Univariate analysis } & \multicolumn{2}{|c|}{ Multivariate analysis } \\
\hline & 5-year CSS (\%) & $P$ value & HR $(95 \% \mathrm{CI})$ & $P$ value \\
\hline Age & & $<0.001$ & & \\
\hline$\leq 60$ & 34.2 & & Reference & \\
\hline$>60$ & 25.1 & & $1.393(1.211-1.603)$ & $<0.001$ \\
\hline Sex & & 0.600 & NI & \\
\hline Male & 31.3 & & & \\
\hline Female & 28.5 & & & \\
\hline Marital status & & $<0.001$ & & \\
\hline Unmarried & 22.9 & & Reference & \\
\hline Married & 34.6 & & $0.735(0.636-0.849)$ & $<0.001$ \\
\hline Unknown & / & & / & \\
\hline Race & & 0.259 & NI & \\
\hline White & 29.0 & & & \\
\hline Black & 35.7 & & & \\
\hline Others & / & & & \\
\hline Serum CEA & & $<0.001$ & & \\
\hline Elevated & 22.9 & & Reference & \\
\hline Normal & 39.3 & & $0.626(0.512-0.746)$ & $<0.001$ \\
\hline Unknown & / & & / & \\
\hline Histologic type & & 0.001 & $\mathrm{NI}$ & \\
\hline Adenocarcinoma & 30.8 & & & \\
\hline Mucinous adenocarcinoma & 29.5 & & & \\
\hline Signet ring cell carcinoma & 6.3 & & & \\
\hline Others & 30.4 & & & \\
\hline Differentiation & & $<0.001$ & Reference & \\
\hline Well & 22.8 & & $0.855(0.611-1.197)$ & 0.362 \\
\hline Moderate & 32.8 & & $1.397(0.984-1.984)$ & 0.061 \\
\hline Poor and undifferentiated & 21.4 & & $0.956(0.638-1.433)$ & 0.827 \\
\hline Unknown & / & & / & \\
\hline Tumor size & & 0.954 & NI & \\
\hline$<5 \mathrm{~cm}$ & 31.1 & & & \\
\hline$\geq 5 \mathrm{~cm}$ & 32.0 & & & \\
\hline Unknown & / & & & \\
\hline T stage & & 0.955 & NI & \\
\hline $\mathrm{T} 1+\mathrm{T} 2$ & 34.7 & & & \\
\hline $\mathrm{T} 3+\mathrm{T} 4$ & 29.6 & & & \\
\hline
\end{tabular}

\begin{tabular}{lllll}
\hline Variable & \multicolumn{2}{l}{ Univariate analysis } & \multicolumn{2}{l}{ Multivariate analysis } \\
\cline { 2 - 6 } & 5 -year CSS $(\%)$ & $P$ value & HR $(95 \% \mathrm{CI})$ & $P$ value \\
\hline Unknown & $/$ & 0.877 & $\mathrm{NI}$ & \\
N stage & 34.9 & & & \\
$\mathrm{~N} 0$ & 29.8 & & & \\
$\mathrm{~N}+$ & $/$ & & & \\
Unknown & & $<.001$ & & \\
Surgery of metastases & 28.6 & & Reference & \\
No & 36.3 & & $0.606(0.498-0.736)$ & $<0.001$ \\
Yes & $/$ & & $/$ & \\
Unknown & & $<0.001$ & & \\
Radiation sequence & 26.8 & & Reference & \\
After surgery & 33.3 & & $0.820(0.712-0.945)$ & $\mathbf{0 . 0 0 6}$ \\
Before surgery & & & &
\end{tabular}

NI: not included in multivariate survival analysis.

Huh et al [11] reported the outcomes of 69 patients received chemoradiotherapy and 71 patients did not. They found that preoperative radiotherapy improved local control of metastatic rectal cancer while postoperative radiotherapy did not. Similarly, Fossum et al [12] also observed a decreased local recurrence rate for patients who received neoadjuvant radiotherapy compared with those who did not receive radiotherapy. In this study, local recurrence was observed in $26 \%$ patients who did not receive radiotherapy, while no local recurrence developed in those who received neoadjuvant radiotherapy. Neoadjuvant radiotherapy demonstrated excellent value for local control in stage IV rectal cancer. However, survival benefit was not observed in the two independent studies. On the contrary, Kim et al [13] evaluated the oncological effects of neoadjuvant 
radiotherapy versus pure postoperative chemotherapy in 86 patients with rectal cancer and synchronous liver metastasis receiving TME procedure and found that neoadjuvant chemoradiotherapy did not improve local control and overall survival. The local recurrence rates were $10 \%$ in neoadjuvant chemoradiotherapy group and 15.2\% in postoperative systemic chemotherapy without radiotherapy group and did not reach a significant difference. Another retrospective study enrolled 109 patients with metastatic rectal adenocarcinoma who underwent definitive primary resection [14]. Compared with patients treated with perioperative chemotherapy alone, no differences were found in terms of local recurrence or overall survival in those who received preoperative radiotherapy. Taken together, the potential advantages of preoperative radiotherapy are hard to determine decisively from these studies. Obviously, a limitation hidden in the above studies is small sample size. Our study eliminates the limitation by analyzing a large-population based database and provides the convincing evidence for the significance of neoadjuvant radiotherapy in resectable metastatic rectal cancer. Further well-designed prospective study should be initiated to explore the optimal therapeutic strategy for these patients and determine which subgroups will benefit the most from this protocol.

It is well documented that distant disease may have a decisive role in prognosis. The resection of distant lesion has been added in our PSM model to reduce the selection bias. Our present study also demonstrated that metastatectomy were significant prognostic factors for CSS in metastatic rectal cancer. In stage IV colorectal cancer, the value of surgery has been well established $[15,16]$. Lin et al [17] reported that concurrent chemoradiotherapy followed by resection of metastases converts to survival benefit in metastatic rectal cancer. These results indicated that neoadjuvant chemoradiotherapy and metastatectomy should be taken into consideration for curative intent.

However, there are several inevitable limitations in our present study. The main deficiency is that the data of systemic chemotherapy is unavailable. Although chemotherapy has a critical effect on prognosis of metastatic rectal cancer patients, the chemotherapy regiments and course of treatment are missing in SEER database. Similarly, neither of BMI nor inflammation index which may affect the prognosis are available. Further, despite we employed the propensity score to minimize selection bias, some unknown confounders remain unnoticeable due to its retrospective nature. Moreover, because preoperative radiotherapy may result in tumor down-sizing and down-staging, inclusion of tumor size, $\mathrm{T}$ stage and $\mathrm{N}$ stage in the PSM model underestimated treatment effect in the preoperative radiotherapy group. A higher percentage of patients with elevated serum CEA level in preoperative radiotherapy group enhanced this effect further.

Despite the above limitations, the relative large sample size, minimal selection bias and sufficient follow-up provide valuable evidence to optimize the therapeutic protocols for resectable metastatic rectal cancer.

In conclusion, for stage IV rectal adenocarcinoma cancer patients received definitive primary resection surgery, we recommend consideration of neoadjuvant radiotherapy instead of postoperative radiotherapy.

\section{Acknowledgments}

This work was supported by the National Natural Science Foundation of China (Grant NO. 81772599; NO. 81702353) and Shanghai Municipal Natural Science Foundation (17ZR1406400). The funders had no role in the study design, data collection and analysis, decision to publish, or preparation of the manuscript.

\section{Competing Interests}

The authors have declared that no competing interest exists.

\section{References}

1. Colorectal Cancer Collaborative G. Adjuvant radiotherapy for rectal cancer: a systematic overview of 8,507 patients from 22 randomised trials. Lancet. 2001; 358: 1291-304.

2. Glimelius BL. The role of preoperative and postoperative radiotherapy in rectal cancer. Clin Colorectal Cancer. 2002; 2: 82-92.

3. Sauer R, Becker H, Hohenberger W, Rodel C, Wittekind C, Fietkau R, et al. Preoperative versus postoperative chemoradiotherapy for rectal cancer. N Engl J Med. 2004; 351: 1731-40.

4. Swedish Rectal Cancer T, Cedermark B, Dahlberg M, Glimelius B, Pahlman L, Rutqvist LE, et al. Improved survival with preoperative radiotherapy in resectable rectal cancer. N Engl J Med. 1997; 336: 980-7

5. Wagman R, Minsky BD, Cohen AM, Guillem JG, Paty PP. Sphincter preservation in rectal cancer with preoperative radiation therapy and coloanal anastomosis: long term follow-up. Int J Radiat Oncol Biol Phys. 1998; 42: 51-7.

6. Madoff RD. Chemoradiotherapy for rectal cancer--when, why, and how? N Engl J Med. 2004; 351: 1790-2.

7. Glynne-Jones R, Wyrwicz L, Tiret E, Brown G, Rodel C, Cervantes A, et al. Rectal cancer: ESMO Clinical Practice Guidelines for diagnosis, treatment and follow-up. Ann Oncol. 2018; 29: iv263.

8. Tong D, Liu F, Li W, Zhang W. The impacts of surgery of the primary cancer and radiotherapy on the survival of patients with metastatic rectal cancer. Oncotarget. 2017; 8: 89214-27.

9. Takada T, Tsutsumi S, Takahashi R, Ohsone K, Tatsuki H, Suto T, et al. Control of primary lesions using resection or radiotherapy can improve the prognosis of metastatic colorectal cancer patients. J Surg Oncol. 2016; 114: 75-9.

10. Lee JH, Jo IY, Lee JH, Yoon SC, Kim YS, Choi BO, et al. The role of postoperative pelvic radiation in stage IV rectal cancer after resection of primary tumor. Radiat Oncol J. 2012; 30: 205-12.

11. Huh JW, Kim HC, Park HC, Choi DH, Park JO, Park YS, et al. Is Chemoradiotherapy Beneficial for Stage IV Rectal Cancer? Oncology. 2015; 89: 14-22.

12. Fossum CC, Alabbad JY, Romak LB, Hallemeier CL, Haddock MG, Huebner $\mathrm{M}$, et al. The role of neoadjuvant radiotherapy for locally-advanced rectal cancer with resectable synchronous metastasis. J Gastrointest Oncol. 2017; 8: 650-8

13. Kim SH, Kim JH, Jung SH. Comparison of oncologic outcomes of metastatic rectal cancer patients with or without neoadjuvant chemoradiotherapy. Int J Colorectal Dis. 2015; 30: 1193-9. 
14. Manyam BV, Mallick IH, Abdel-Wahab MM, Reddy CA, Remzi FH, Kalady $\mathrm{MF}$, et al. The Impact of Preoperative Radiation Therapy on Locoregional Recurrence in Patients with Stage IV Rectal Cancer Treated with Definitive Surgical Resection and Contemporary Chemotherapy. J Gastrointest Surg. 2015; 19: 1676-83.

15. Luo D, Liu Q, Yu W, Ma Y, Zhu J, Lian P, et al. Prognostic value of distant metastasis sites and surgery in stage IV colorectal cancer: a population-based study. Int J Colorectal Dis. 2018; 33:1241-49.

16. Ahmed S, Leis A, Chandra-Kanthan S, Fields A, Reeder B, Iqbal N, et al. Surgical Management of the Primary Tumor in Stage IV Colorectal Cancer: A Confirmatory Retrospective Cohort Study. J Cancer. 2016; 7: 837-45.

17. Lin JK, Lee LK, Chen WS, Lin TC, Jiang JK, Yang SH, et al. Concurrent chemoradiotherapy followed by metastasectomy converts to survival benefit in stage IV rectum cancer. J Gastrointest Surg. 2012; 16: 1888-96. 